LU TP 01-13

LUNFD6/(NFFL-7194)2001

\title{
Hadronization corrections to helicity components of the fragmentation function
}

\author{
T. Sjöstrand ${ }^{1}$, O. Smirnova ${ }^{2 *}$ and Ch. Zacharatou Jarlskog ${ }^{2}$ \\ ${ }^{1}$ Department of Theoretical Physics, Lund University, Sölvegatan 14A, S 22362 Lund, Sweden \\ ${ }^{2}$ Department of Elementary Particle Physics, Lund University, P.O.Box 118, S 22100 Lund, \\ Sweden
}

\begin{abstract}
In the hadronic decays of $Z^{0}$, gluon emission leads to the appearance of the longitudinal component of the fragmentation function, $F_{L}$. Measurement of $F_{L}$ and the transverse component, $F_{T}$, could thus provide an insight into the gluon fragmentation function. However, hadronization corrections at low $x$ can be significant. Here we present a method of accounting for such corrections, using the JETSET event generator as illustration.
\end{abstract}

\section{Introduction}

Studies of fragmentation functions have always been important, since these distributions can not be predicted theoretically, but only be measured experimentally, and consecutively be described by phenomenological models. Hadronic decays of $\gamma^{*} / Z^{0}$ provide a particularly convenient set of events for analysis and interpretation. Helicity components of the fragmentation function, measured in such events, can be used in various QCD studies, e.g., the extraction of the gluon fragmentation function, and the evaluation of $\alpha_{s}$. However, existing theoretical calculation being restricted to the perturbative region, hadronization corrections must be taken into account. In what follows, methods for applying such corrections using the JETSET event generator 值 as an example, will be discussed.

Consider the angular distribution in the process $e^{+} e^{-} \rightarrow \gamma^{*} / Z^{0} \rightarrow q \bar{q}$ in its rest frame. Assuming that the final quark and antiquark are not charge-tagged, i.e. that

*On leave from: JINR, 141980 Dubna, Russia 
the forward-backward asymmetry is not accessed, the cross section can be written as [2]

$$
\frac{\mathrm{d} \sigma}{\mathrm{d}(\cos \theta)}=\frac{3}{8}\left(1+\cos ^{2} \theta\right) \sigma_{\mathrm{T}}+\frac{3}{4} \sin ^{2} \theta \sigma_{\mathrm{L}} .
$$

Here $\sigma_{\mathrm{T}}\left(\sigma_{\mathrm{L}}\right)$ is the cross section associated with a transverse (longitudinal) gauge boson polarization state with respect to the $q \bar{q}$ axis, and $\theta$ is the polar angle of a particle with respect to the incoming lepton axis. To lowest order, only mass effects contribute to a non-vanishing $\sigma_{\mathrm{L}}$, but only for the vector part of the cross section, and there only with a coefficient $\sigma_{\mathrm{L}} / \sigma_{\mathrm{T}}=2 m_{q}^{2} / E_{\mathrm{cm}}^{2}$. Even for the $b$ quarks this gives a negligible $\sigma_{\mathrm{L}}$ contribution at the energies around the $Z^{0}$ peak. Therefore, $\sigma_{\mathrm{L}}$ effectively starts in $\mathcal{O}\left(\alpha_{\mathrm{s}}\right)$ of perturbation theory, associated with the emission of gluons.

Since partons are not directly observable, one may define a hadron-level analogue of Eq.(11) [3],

$$
\frac{\mathrm{d}^{2} \sigma^{\mathrm{h}}}{\mathrm{d} x \mathrm{~d}(\cos \theta)}=\frac{3}{8}\left(1+\cos ^{2} \theta\right) \frac{\mathrm{d} \sigma_{\mathrm{T}}}{\mathrm{d} x}+\frac{3}{4} \sin ^{2} \theta \frac{\mathrm{d} \sigma_{\mathrm{L}}}{\mathrm{d} x} .
$$

Here $x$ would preferably be associated with the energy fraction taken by a hadron, $x_{E}=2 E / E_{\mathrm{cm}}$, so that $\sum x_{E}=2$ in each event. Experimentally it is more convenient to use the momentum fraction $x_{p}$. The transverse and longitudinal fragmentation functions are defined by a normalization to the total cross section $\sigma_{\mathrm{tot}}=\sigma_{\mathrm{T}}+\sigma_{\mathrm{L}}$ 涵,

$$
F_{\mathrm{T}}(x)=\frac{1}{\sigma_{\text {tot }}} \frac{\mathrm{d} \sigma_{\mathrm{T}}}{\mathrm{d} x}, \quad F_{\mathrm{L}}(x)=\frac{1}{\sigma_{\text {tot }}} \frac{\mathrm{d} \sigma_{\mathrm{L}}}{\mathrm{d} x} .
$$

The former is dominated by the fragmentation of quark jets, whereas the latter receives a major contribution from gluon fragmentation. Therefore an experimental determination of $F_{\mathrm{L}}(x)$ is a first step towards an extraction of the gluon fragmentation function, alternative to what is offered by more direct methods in three-jet events [5]. Several experimental $F_{\mathrm{L}}(x)$ studies have also been presented [6].

A complication is that hadrons are not moving in the direction of their imagined mother parton. Already in lowest order of perturbation theory, for $q \bar{q}$ two-jet events, nonperturbative hadronization gives an effective $p_{\perp}$ smearing that induces a nonvanishing $F_{\mathrm{L}}(x)$ even where none is expected. Furthermore, the association of a hadron to a single mother parton is not in agreement with our current best understanding of the hadronization process, where it is rather the colour field between a colour-connected pair of partons (a string piece [7], or a cluster [8]) that mediates the hadron production. Therefore the structure of smearing effects may become rather nontrivial. Obviously, the effects are especially important at small $x$, which is also the region where one would hope to have some sensitivity to the gluon fragmentation function. The string picture also casts in doubt the concept of a gluon fragmentation function defined from inclusive quantities, since the string fragmentation of a parton depends on the angles to other colour-connected partons.

The issue of hadronization corrections to fragmentation functions was addressed in [4, 9]. The emphasis was on the $\sigma_{\mathrm{L}}$ that can be extracted from $\int_{0}^{1} F_{\mathrm{L}}(x) x \mathrm{~d} x$ rather than on $F_{\mathrm{L}}(x)$ itself, however. Therefore we here address how hadronization affects $F_{\mathrm{L}}(x)$ (and $F_{\mathrm{T}}(x)$ ). One main conclusion is that a simple smearing approach is not sufficient to describe hadronization effects. Thus it appears impossible to 

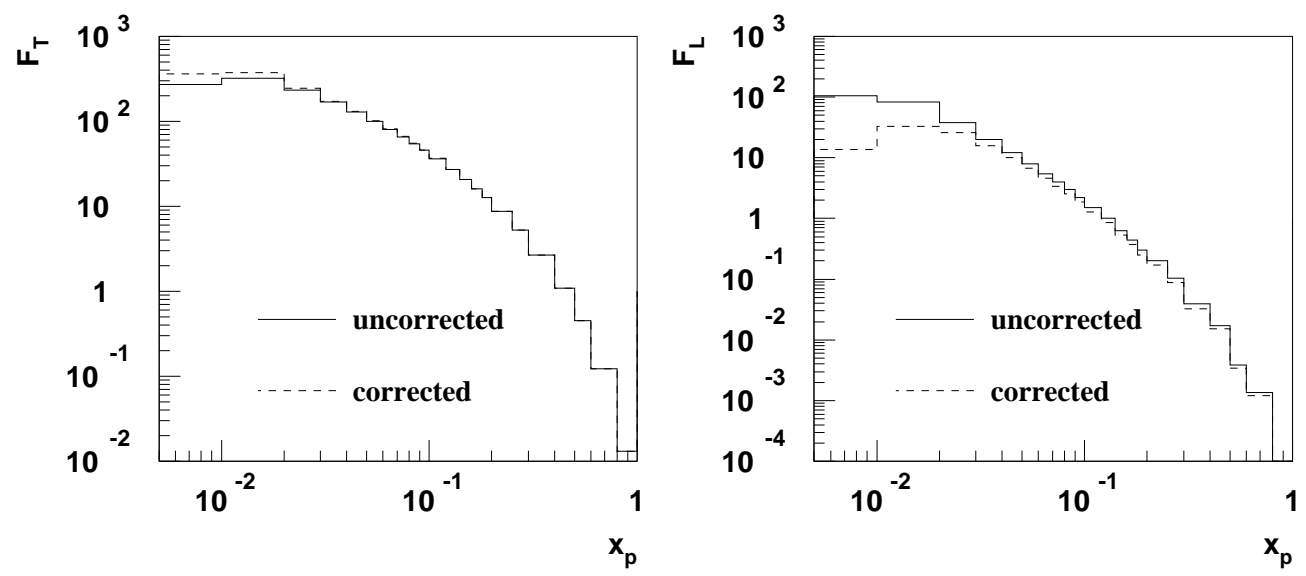

Figure 1: $F_{T}\left(x_{p}\right)$ and $F_{L}\left(x_{p}\right)$ for corrected (smeared) hadron angles (dashed lines) and for uncorrected (non-smeared) hadron angles (solid lines).

define a completely model-independent, hadronization-smearing-corrected $F_{\mathrm{L}}(x)$, that could be used to extract a gluon fragmentation function. We further suggest a correction procedure, based on a cluster search strategy, that should give a less model dependent $F_{\mathrm{L}}(x)$, but at the price of introducing the cluster resolution scale $y$ as a new parameter in the problem.

\section{The simple smearing}

The string model description of $q \bar{q}$ events introduces a Gaussian transverse momentum smearing of primary hadrons, $\propto \exp \left(-p_{\perp}^{2} / 2 \sigma^{2}\right) \mathrm{d}^{2} p_{\perp}$, where $\sigma \approx 0.36 \mathrm{GeV}$ [1, 10]. Many primary hadrons are unstable and decay further; this distorts the original Gaussian spectrum and reduces the average $p_{\perp}$. Since decay products have smaller $p_{\|}$, momentum parallel to the jet axis, some correlation is also introduced between $p_{\perp}$ and $p_{\|}$. Therefore no simple parameterization is proposed, but instead a Monte Carlo simulation with JETSET 7.4 [1] is used to histogram the amount of angular smearing for different $x_{p}$ bins.

It is now assumed that this smearing should be applied both to quark and gluon jets, so that a hadron will not move exactly in the direction of its mother parton. There are obvious shortcomings to equating different kinds of jets, like that gluon jets have a lower energy and do not contain decays of charm and bottom hadrons, but those particular issues only introduce moderate corrections. More severe objections can be raised to the association of hadrons to individual partons, as we will discuss further in the next section, but forget for the moment.

Had particles not been smeared in $p_{\perp}$, but parallel with their parton of origin, the shape of the angular distribution

$$
F\left(x_{p}, \cos \theta\right)=\frac{3}{8}\left(1+\cos ^{2} \theta\right) F_{\mathrm{T}}\left(x_{p}\right)+\frac{3}{4} \sin ^{2} \theta F_{\mathrm{L}}\left(x_{p}\right)
$$


in a bin of $x_{p}$ could be used to extract $F_{\mathrm{T}}\left(x_{p}\right)$ and $F_{\mathrm{L}}\left(x_{p}\right)$ in that bin. The abovementioned smearing will now modify this. The two angular shapes, $(3 / 8)\left(1+\cos ^{2} \theta\right)$ and $(3 / 4) \sin ^{2} \theta$, both normalized to unity, are therefore convoluted with the $x_{p^{-}}$ dependent smearing distributions, characterized by a distribution in the smearing angle $\theta^{\mathrm{sm}}$ and an isotropic azimuthal distribution $\varphi^{\mathrm{sm}}$. That is, a parton at an angle $\theta^{\mathrm{p}}$ will produce a hadron at an angle $\theta^{\mathrm{h}}$, where

$$
\cos \theta^{\mathrm{h}}=\cos \theta^{\mathrm{p}} \cos \theta^{\mathrm{sm}}-\sin \theta^{\mathrm{p}} \sin \theta^{\mathrm{sm}} \cos \varphi^{\mathrm{sm}} .
$$

Data can now be fitted both to the "non-smeared" angular distribution form, Eq.(四), and to the convoluted ("smeared") analogue. The resulting distributions for $F_{T}$ and $F_{L}$, obtained using the JETSET generated events, are shown in Fig. 1. The effect on $F_{T}$ and $F_{L}$ is visible for hadron momenta below $10 \%$ of the beam energy. The low momentum region is affected the most, giving $F_{L}$ values reduced up to one order of magnitude.

\section{Objections to the simple smearing}

The above smearing procedure is correct to lowest order in $\alpha_{\mathrm{s}}$, i.e. it describes how two-jet events can induce a nonvanishing $F_{\mathrm{L}}\left(x_{p}\right)$. We know, however, that hadronization of three-jet events cannot be described in terms of a simple incoherent sum of three $q, \bar{q}$ and $g$ jets. One example is the string/drag effect [11, 12], i.e. that particle production is suppressed in the angular region between the $q$ and $\bar{q}$ and enhanced in the other two regions, well confirmed experimentally [13]. High-momentum hadrons still essentially follow the separate parton directions, but low-momentum ones are significantly affected. These are the ones where the angular smearing effects are large to begin with. It is well-known that the string effect leads to more twojetlike events, e.g. in terms of thrust $T$, than implied by symmetric smearing [14. The reason is to be found in the enhanced production of particles between two colour-connected partons that are close in angle, leading to them seemingly being even closer, e.g. that the opening angle between the reconstructed jets typically is smaller than that between the original partons.

There is a nontrivial topology dependence on string effects, especially when multiple gluon emission is considered. The issue is therefore best studied in an event generator, by comparing angular distributions on the parton ( $i=\mathrm{p}$ below) and on the hadron $(i=\mathrm{h})$ level. As a simple measure of the jettiness of events we use $\bar{T}=1-T$, defined on the parton level of each event. The integrated $x_{E}$ spectrum is decomposed as

$$
\begin{aligned}
& \left(\frac{\mathrm{d} \sigma_{\text {tot }}}{\mathrm{d} \bar{T}}\right)^{-1} \int_{0}^{1} \frac{\mathrm{d}^{3} \sigma^{i}}{\mathrm{~d} \bar{T} \mathrm{~d} x_{E} \mathrm{~d}(\cos \theta)} x_{E} \mathrm{~d} x_{E}= \\
= & \frac{3}{8}\left(1+\cos ^{2} \theta\right) F_{\mathrm{T}}^{i}(\bar{T})+\frac{3}{4} \sin ^{2} \theta F_{\mathrm{L}}^{i}(\bar{T}),
\end{aligned}
$$

where the $x_{E}$-weighting ensures a common normalization $F_{\mathrm{T}}^{i}(\bar{T})+F_{\mathrm{L}}^{i}(\bar{T})=2$ at parton and hadron level (values of $F_{\mathrm{T}}^{i}$ and $F_{\mathrm{L}}^{i}$ most conveniently are obtained by weighting each particle with an appropriate angular factor 那). Then $\Delta F_{\mathrm{L}}(\bar{T})=$ $F_{\mathrm{L}}^{\mathrm{h}}(\bar{T})-F_{\mathrm{L}}^{\mathrm{p}}(\bar{T})$ is a simple measure of the hadronization impact on $F_{\mathrm{L}}$. This quantity 


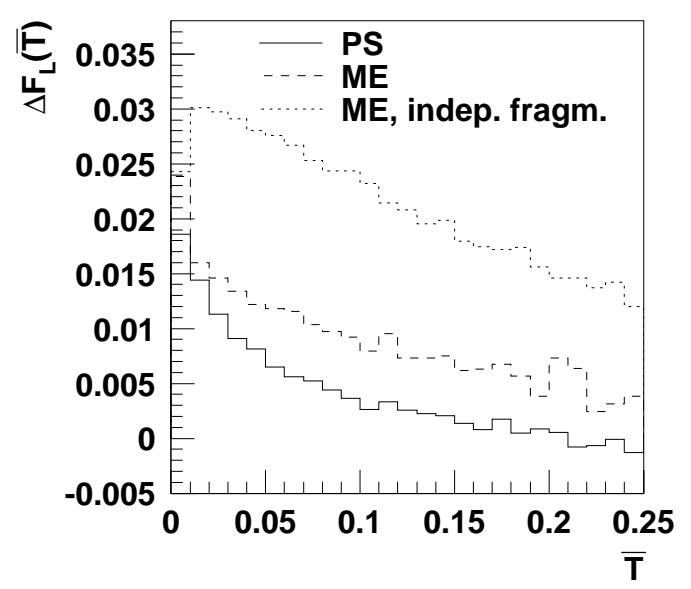

Figure 2: Event-by-event hadronization corrections $\Delta F_{\mathrm{L}}(\bar{T})=F_{\mathrm{L}}^{\mathrm{h}}(\bar{T})-F_{\mathrm{L}}^{\mathrm{p}}(\bar{T})$ for different simulations: solid line corresponds to JETSET 7.4 PS, dashed-JETSET 7.4 ME (both using the string fragmentation), and dotted - JETSET 7.4 ME with independent fragmentation scheme.

is shown in Fig. 2, for one realistic simulation and two toy ones, for $u \bar{u}$ events at $91.2 \mathrm{GeV}$. In the realistic case, a parton shower is used to generate multiparton configurations, followed by string fragmentation. The shower develops down to a cut-off scale $Q_{0} \approx 1 \mathrm{GeV}$, so that also events in the first bin, $\bar{T}<0.01$, can contain some gluons. The other two histograms are based on $\mathcal{O}\left(\alpha_{\mathrm{s}}\right)$ matrix elements, where only 2- and 3-parton configurations are generated, with a cut $\bar{T}>0.01$ on the latter to avoid the singularities of the 3-parton matrix element. Thus the first bin here represents pure 2-parton events. While one simulation is again based on string fragmentation, the other assumes isotropic smearing around the jet axes, basically the independent fragmentation scheme of Hoyer et al. [15, 14]. (The same fragmentation parameters, tuned to the shower model, have been used in all three cases. A retuning of parameters for the $\mathcal{O}\left(\alpha_{\mathrm{s}}\right)$ simulations would have given a larger nonperturbative $p_{\perp}$ width $\sigma$ to cover for the lack of perturbative gluons, and so would have implied even larger fragmentation smearing.)

The isotropic smearing is, as expected, giving a rather constant hadronization correction $\Delta F_{\mathrm{L}}(\bar{T})$. There is some jump up in going from two to three jets that are smeared, followed by a slow but steady drop with $\bar{T}$, since the longitudinal component itself is increasing in importance with $\bar{T}$ and therefore gives an increasing hadronization smearing of the longitudinal component onto the transverse one rather than only the other way around. By contrast, the string fragmentation provides a much steeper drop of $\Delta F_{\mathrm{L}}$ with $\bar{T}$, kicking in immediately when going from two to three partons, and enhanced in the shower simulation relative to the simpler $\mathcal{O}\left(\alpha_{\mathrm{s}}\right)$ one. At large $\bar{T}$ the overall hadronization correction can even turn negative. Averaging over the $\bar{T}$ spectrum (with mean value $\langle\bar{T}\rangle \approx 0.05$ ), we conclude that the typical hadronization smearing contribution is only about a third of the naively expected one, as obtained from two-parton results. (Qualitatively this agrees with 
and probably explains a similar observation in [4] of smaller-than-expected hadronization corrections when using HERWIG [16].) That is, if hadronization corrections are viewed as a power series in $\alpha_{\mathrm{s}}$, the $\mathcal{O}\left(\alpha_{\mathrm{s}}\right)$ term is of opposite sign and almost as big as the $\mathcal{O}(1)$ one.

It should be remembered, however, that this is integrated over all $x_{E}$, and that we have no similar way of addressing results in specific $x$ bins, since the parton and hadron $x$ spectra are quite different. Thus it is likely that the $F_{\mathrm{L}}\left(x_{p}\right)$ derived in the previous section is an underestimation, just like an $F_{\mathrm{L}}\left(x_{p}\right)$ found without any smearing corrections is likely to be an overestimation, but it appears impossible to find the "correct" $F_{\mathrm{L}}\left(x_{p}\right)$ without making detailed assumptions about the hadronization process.

\section{Clustering}

Given the problems with the above smearing recipe, we introduce a new strategy, based on the clustering approach. In a nutshell, we propose to rotate all hadrons to the direction of the cluster they belong to, as an approximate way of removing hadronization smearing effects. Only thereafter is $F_{\mathrm{L}}(x)$ extracted from this modified $\cos \theta$ distribution. The strategy is explained further in the following.

In clustering algorithms, nearby hadrons are combined to form clusters/jets, in a way that should reflect the underlying partonic state, to some approximation. The combination process is controlled by (at least) one separation parameter, call it $y_{\min }$, such that the final state contains no pair of clusters closer to each other than that. Clustering algorithms can be applied also to a partonic state, and here $y_{\text {min }}$ provides a regularization of soft and collinear divergences in the perturbative cross sections. It is then meaningful to calculate the distribution of partons at a factorization scale $\mu^{2}=y_{\min } E_{\mathrm{cm}}^{2}$, and define scale-dependent fragmentation functions parameterizing the subsequent soft-perturbative and nonperturbative hadronization. The latter should obey standard QCD evolution equations, starting from some unknown nonperturbative form at a low reference scale.

Over the years many cluster algorithms have been proposed [17], each with its strengths and weaknesses. In this article we adopt the Durham one [18], which is a standard for many perturbative calculations. The distance measure between two clusters $i$ and $j$ is

$$
y_{i j}=\frac{2 \min \left(E_{i}^{2}, E_{j}^{2}\right)\left(1-\cos \theta_{i j}\right)}{E_{\mathrm{vis}}^{2}},
$$

so that $\sqrt{y_{i j}}$ roughly corresponds to the relative transverse momentum, scaled to the total visible energy $E_{\mathrm{vis}}$ ( $=E_{\mathrm{cm}}$ for an ideal detector).

If we begin by considering a simple $q \bar{q}$ event, it should reconstruct back to two clusters, unless $y_{\min }$ has been chosen very small. Since the momentum of a cluster is given by the vector sum of its constituent hadrons, it would resum opposite and compensating $p_{\perp}$ kicks imparted to hadrons in the fragmentation process. The cluster direction should therefore be a better measure of the $q \bar{q}$ axis than that provided by the individual hadron momenta. It is the angular distribution of this axis that relates back to the polarization character of the $\gamma^{*} / Z^{0} \rightarrow q \bar{q}$ decay, and that we want to be reflected in our extracted $F_{\mathrm{L}}(x)$ and $F_{\mathrm{T}}(x)$. Therefore it would 
be an improvement to rotate all hadrons in a cluster to sit along the cluster direction. That is, the $\theta$ of a hadron is redefined while its $x$ value is unchanged.

At this level there is no contradiction with the smearing approach studied earlier. Then we smeared the simple partonic angular shapes to arrive at realistic hadronic ones to compare with data, now we un-smear the hadronic angles to approach the simple partonic distributions. There is one advantage, however: the clustering approach is not sensitive to the width of the $p_{\perp}$ distribution, i.e. the $\sigma$ parameter, unlike the smearing procedure. Of course, the $p_{\perp}$ width still affects the typical error between the $q \bar{q}$ and cluster axes.

When considering multijet production, the $y_{\min }$ choice does become relevant, with $\mu^{2}=y_{\min } E_{\mathrm{cm}}^{2}$ acting as a factorization scale, as noted above. For a large $y_{\min }$ all activity is clustered into the two quark jets, and neither the gluon structure nor $F_{\mathrm{L}}(x)$ would be probed. For $y_{\min } \rightarrow 0$ each hadron or parton is a cluster unto itself, and we are back at the starting point. So obviously some intermediate scale is to be preferred. Given that the typical hadronization $p_{\perp}$ width is $\sim 0.4 \mathrm{GeV}$, with a tail to larger values, one would conclude that clustering up to $p_{\perp} \sim 1 \mathrm{GeV}$ would be a sensible minimum to eliminate the bulk of the hadronization $p_{\perp}$ smearing. At the $Z^{0}$ peak this translates into $y_{\text {min }} \gtrsim 0.0001$. In the upper end, we want to stay with a picture of multiple gluon emission as the norm, i.e. retain $F_{\mathrm{L}}(x)$ as an inclusive quantity, in order not to overlap with traditional studies of gluon jets in exclusive three-jet events. Since the average number of clusters per event is three for $y_{\text {min }} \approx 0.0025$, we conclude that $0.0001 \lesssim y_{\text {min }} \lesssim 0.0025$ is a reasonable range, over which to study a scale-dependent $F_{\mathrm{L}}(x, \mu)$.

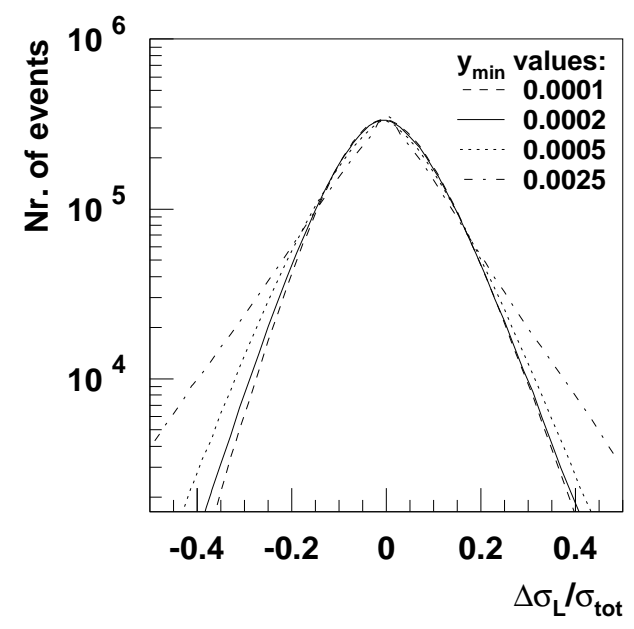

Figure 3: Event-by-event hadronization corrections $\Delta \sigma_{\mathrm{L}} / \sigma_{\text {tot }}=\left(\sigma_{\mathrm{L}}^{h}-\sigma_{\mathrm{L}}^{p}\right) / \sigma_{\text {tot }}$ for different $y_{\text {min }}$ scales (JETSET 7.4 PS and string fragmentation).

In Fig. 3, the distribution of event-by-event $x_{E}$-weighted and -integrated hadronization corrections $\Delta \sigma_{\mathrm{L}} / \sigma_{\text {tot }}=\left(\sigma_{\mathrm{L}}^{h}-\sigma_{\mathrm{L}}^{p}\right) / \sigma_{\text {tot }}$ is shown for some different $y_{\text {min }}$ scales, for events generated with parton showers and string fragmentation (superscripts $h$ and $p$ stand for hadron and parton level, respectively). We note the significant width of these distributions, showing that event-by-event fluctuations in 
the hadronization process are important and can be of either sign. Even if small by comparison, the mean $\left\langle\Delta \sigma_{\mathrm{L}} / \sigma_{\text {tot }}\right\rangle$ does show a systematic bias, positive for small $y_{\min }$ and negative for large $y_{\min }$. That is, at small $y_{\min }$ the hadronization smearing wins over the string effects, while it is the other way around for large $y_{\min }$ - but remember that this is only true when averaging over many events. Nevertheless, one possible criterion for a good choice of $y_{\min }$ would be where the two effects cancel, which then gives $y_{\min } \approx 0.0002$, i.e. $\mu \approx 1.3 \mathrm{GeV}$. While a sensible reference value, one should not take this particular value too seriously, since it is for one specific model, and for one specific set of model parameters. Somewhat different parameter values, like for the parton shower cut-off $Q_{0} \approx 1 \mathrm{GeV}$, defining the parton level of the events studied, could lead to slightly different "preferred" $\mu$ values.

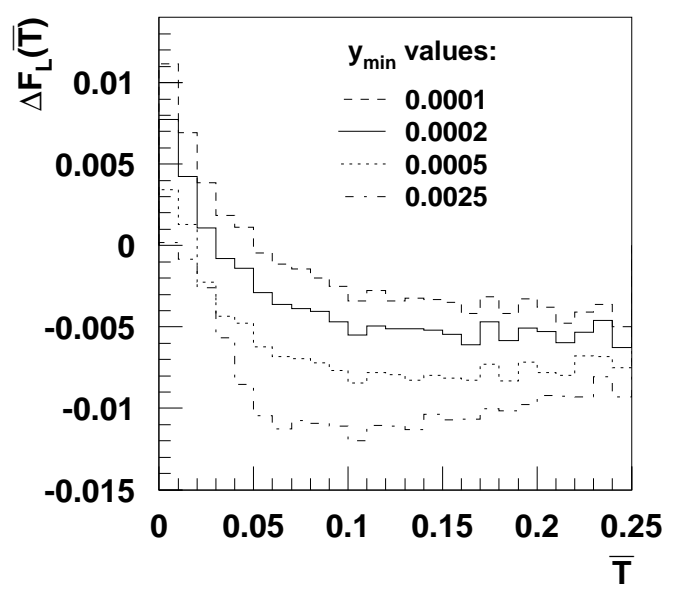

Figure 4: Hadronization corrections $\Delta F_{\mathrm{L}}(\bar{T})=F_{\mathrm{L}}^{\mathrm{h}}(\bar{T})-F_{\mathrm{L}}^{\mathrm{p}}(\bar{T})$ for different $y_{\min }$ scales (JETSET 7.4 PS and string fragmentation).

It is important to note that we here have been considering the $x$-integrated quantity. This is of relevance if one e.g. would like to extract an $\alpha_{\mathrm{s}}$ from an $\sigma_{\mathrm{L}}$ measurement, and so not uninteresting. For the purpose of determining the differential $x$ distribution, $F_{\mathrm{L}}(x)$, however, one would have reason to fear that any bias could have an $x$ dependence that would not be caught. In the string model, a string piece connecting two partons is boosted by an increasing velocity vector as the relative opening angle between the partons is decreased, and so the string effects spread upwards to larger $x$ values. A warning signal is then that $\Delta F_{\mathrm{L}}$ does depend quite significantly on $\bar{T}$, Fig. $\mathbb{\theta}$, i.e. clustering does not reduce the $\bar{T}$ dependence noted in Fig. 2, but mainly shifts the overall level. Since $\bar{T}$ probes the topology of events, we also do expect this topology to reflect itself in an $x$ dependence of hadronization corrections. As in the previous studies, this dependence is then likely to show up mainly in the lower end of the $x$ range. At larger $x$, hadrons are rather well aligned with the jet axes, so, even with $x$-weighting, the few particles out there give a small contribution to the $\left\langle\Delta F_{\mathrm{L}}\right\rangle$. In Fig 5, the relative difference between the inclusive $F_{L}\left(x_{p}\right)$ and $F_{L}^{\text {cluster }}\left(x_{p}\right)$, obtained by replacing hadron angles with cluster angles for different $y_{\min }$ values, is shown. It is clearly seen, indeed, that 
the hadronization corrections are only important at low $x_{p}$, unless $y_{\min }$ is chosen too high. For the transverse component of the fragmentation function, $F_{T}\left(x_{p}\right)$, corrections have the same absolute amplitude but the opposite sign.

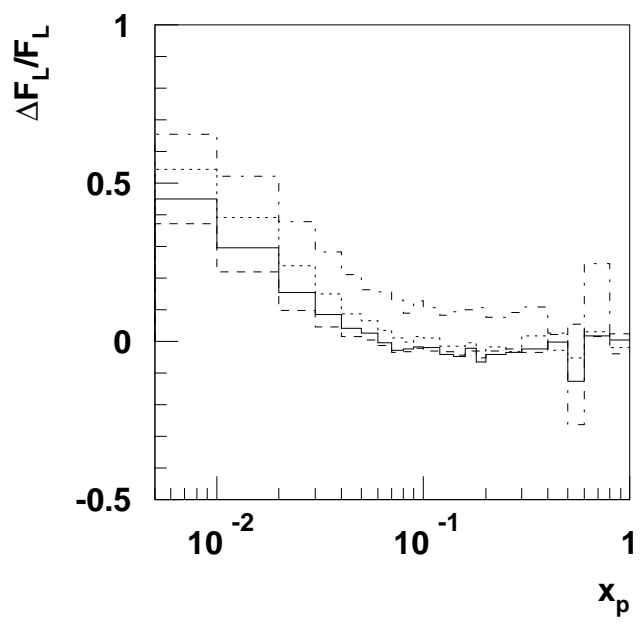

Figure 5: Ratio $\Delta F_{\mathrm{L}} / F_{\mathrm{L}}=\left(F_{\mathrm{L}}-F_{\mathrm{L}}^{\text {cluster }}\right) / F_{\mathrm{L}}$ for various $y_{\text {min }}$ values: dashed line corresponds to $y_{\min }=0.0001$, solid $-y_{\min }=0.0002$, dotted $-y_{\min }=0.0005$ and dash-dotted $y_{\text {min }}=0.0025$.

On the up side, the string effect has its perturbative equivalent in the colour dipole [12]. That is, asymmetries also exist in the production of soft gluons around the direction of the harder partons of an event. Such soft parton emission, below the cut-off scale $Q_{0} \approx 1 \mathrm{GeV}$ we have used, would thus largely fill in the same regions as the nonperturbative hadron production, and with the same topology dependence. If one takes Local Parton-Hadron Duality [19] seriously, this equivalence should come very close. Our proposed strategy, to reset the $\theta$ angle of particles to that of the cluster they belong to, would be applicable also to such perturbatively calculated parton topologies.

\section{Summary}

The coherence phenomenon [20] kills the concept of gluon fragmentation functions that can be defined independently of the environment they are found in. The "hump-backed" shape of inclusive $x$ spectra [19, 21] is an excellent illustration: by coherence the multiplication of partons/hadrons at small $x$ is much less than if the hard partons could radiate/hadronize independently. The immediate consequence is that the expected "softer gluon than quark jets" picture is difficult to test. This impacts both on studies of gluon jets directly in identified three-jet events and indirectly via $F_{\mathrm{L}}(x)$. In this article we have illustrated some of these issues for the latter observable.

It appears safe to conclude that a straightforward extraction of $F_{\mathrm{L}}(x)$ from hadron angular distributions exaggerates the rate of particles at small $x$ that should be attributed to gluon jets, since even the hadronization of pure $q \bar{q}$ events induces a 
'false' $F_{\mathrm{L}}(x)$ by $p_{\perp}$ smearing. We have also here shown that a symmetric smearing around jet axes introduces a bias in the other direction, since it misses important string/drag effects that tend to make three-jet events more two-jetlike. In summary, there is no model-independent extraction of a unique $F_{\mathrm{L}}(x)$, especially not at small $x$ values.

We therefore propose to introduce a scale-dependent quantity $F_{\mathrm{L}}\left(x, \mu^{2}\right)$. Particles are clustered, e.g. with the Durham algorithm, and thereafter assigned the $\theta$ angle of the cluster they belong to, while retaining their $x$ value. Thus $\mu^{2}$ sets an 'unsmearing' scale, below which $p_{\perp}$ fluctuations are killed. We find that a $\mu \approx 1.3 \mathrm{GeV}$ gives opposite and compensating $p_{\perp}$ smearing and string effects in JETSET simulations. While the exact number certainly is model-dependent, the order is a sensible one, given that the average hadronization $p_{\perp}$ is of the order of $0.4 \mathrm{GeV}$. If this then sets a reasonably lower limit, an upper one is related to the desire to stay away from the region of exclusive two- or three-jet events. Over an intermediate range, one could imagine several experimental determinations providing the scale dependence. We also remind that, so far, our studies have only been intended for LEP1 energies. Coverage of a wider energy range, e.g. at LEP2, introduces $s$ as a further scale of the process and allows more differential tests.

\section{References}

[1] T. Sjöstrand, Computer Phys. Commun. 82 (1994) 74.

[2] A. C. Hirschfeld and G. Kramer, Nucl. Phys. B74 (1974) 211.

[3] G. Altarelli et al., Nucl. Phys. B160 (1979) 301; Phys. Rep. 81, (1982) 1.

[4] P. Nason and B. R. Webber, Nucl. Phys. B421 (1994) 473, erratum B480 (1996) 755.

[5] OPAL Coll., G. Abbiendi et al., Eur. Phys. J. C11 (1999) 217;

DELPHI Coll., P. Abreu et al., Eur. Phys. J. C13 (2000) 573;

ALEPH Coll., R. Barate et al., Eur. Phys. J. C17 (2000) 1.

[6] TASSO Coll., R. Brandelik et al., Phys. Lett. B114 (1982) 65;

OPAL Coll., R. Akers et al., Z. Phys. C68 (1995) 203;

ALEPH Coll., D. Buskulic et al., Phys. Lett. B357 (1995) 487;

DELPHI Coll., P. Abreu et al., Eur. Phys. J. C6 (1999) 19.

[7] B. Andersson, G. Gustafson, G. Ingelman and T. Sjöstrand, Phys. Rep. 97 (1983) 31 ;

B. Andersson, The Lund Model (Cambridge University Press, 1998).

[8] B. R. Webber, Nucl. Phys. B238 (1984) 492.

[9] Yu. L. Dokshitzer, B. R. Webber, Phys. Lett. B352 (1995) 451;

M. Dasgupta, B. R. Webber, Nucl. Phys. B484 (1997) 247;

M. Beneke, V. M. Braun, L. Magnea, Nucl. Phys. B497 (1997) 297.

[10] I. G. Knowles et al., in Physics at LEP2, eds. G. Altarelli, T. Sjöstrand and F. Zwirner, CERN 96-01, Vol. 2, p. 103

[11] B. Andersson, G. Gustafson and T. Sjöstrand, Phys. Lett. 94B (1980) 211 
[12] Ya. I. Azimov, Yu. L. Dokshitzer, V. A. Khoze and S. I. Troyan, Phys. Lett. 165B (1985) 147

[13] JADE Coll., W. Bartel et al., Z. Phys. C21 (1983) 37;

OPAL Coll., R. Akers et al., Z. Phys. C68 (1995) 531;

DELPHI Coll., P. Abreu et al., Z. Phys. C70 (1996) 179;

ALEPH Coll., R. Barate et al., Phys. Rep. 294 (1998) 1.

[14] T. Sjöstrand, Z. Phys. C26 (1984) 93.

[15] P. Hoyer, P. Osland, H. G. Sander, T. F. Walsh and P. M. Zerwas, Nucl. Phys. B161 (1979) 349.

[16] G. Marchesini, B. R. Webber, G. Abbiendi, I. G. Knowles, M. H. Seymour and L. Stanco, Computer Phys. Commun. 67 (1992) 465.

[17] for a review see e.g. S. Moretti, L. Lönnblad and T. Sjöstrand, J. High Energy Phys. 08 (1998) 001.

[18] S. Catani, Yu. L. Dokshitzer, M. Olsson, G. Turnock and B. R. Webber, Phys. Lett. B269 (1991) 432.

[19] Ya. I. Azimov, Yu. L. Dokshitzer, V. A. Khoze and S. I. Troyan, Z. Phys. C27 (1985) 65, Z. Phys. C31 (1986) 213.

[20] A. H. Mueller, Phys. Lett. 104B (1981) 161;

B. I. Ermolaev and V. S. Fadin, JETP Lett. 33 (1981) 269.

[21] Yu. L. Dokshitzer, V. S. Fadin and V. A. Khoze, Phys. Lett. B115 (1982) 242; Z. Phys. C15 (1982) 325; Z. Phys. C18 (1983) 37;

A. Bassetto, M. Ciafaloni, G. Marchesini and A. H. Mueller, Nucl. Phys. B207 (1982) 189. 